\title{
Diferencias en los tipos y niveles de agresividad en adolescentes víctimas y no víctimas del conflicto armado en Colombia*
}

\author{
Differences in the types and levels of aggression in adolescent \\ victims and non-victims of the armed conflict in Colombia
}

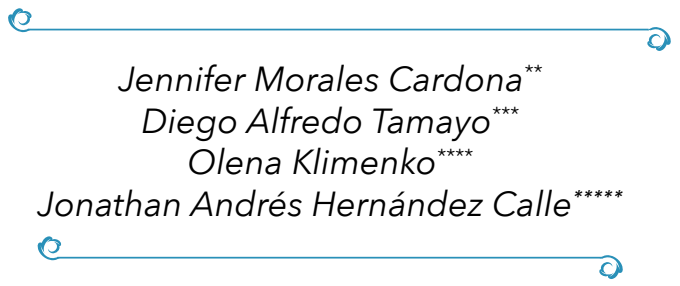

Recibido 20.08. $2018 \bullet-$ Arbitrado 09. 10. $2018 \bullet$ Aprobado 15.11.2018

* Investigación realizada para otra el título de psicólogo, Facultad de Ciencias Sociales, Institución Universitaria de Envigado.

* Psicóloga, Institución Universitaria de Envigado, jennifermoralesc9@gmail. com

*to Psicólogo, Magister en Neurociencias, Docente tiempo completo, Institución Universitaria de Envigado, pditalo@ gmail.com, ORCID: 0000-0001-79968871

Psicóloga, Magister en Ciencias Sociales, Doctora en Psicopedagogía, Docente tiempo completo, Institución Universitaria de Envigado. Colombia, olenak45@gmail.com, ORCID: 00000002-8411-1263

*atur Psicólogo, Magister en Epidemiologia, Docente Institución Universitaria de Envigado, nathancalleqyahoo.es, ORCID:0000-0001-7749-1547

\section{Resumen}

Introducción: Colombia ha sido un país con numerosos precedentes de conflicto armado, el cual ha dejado grandes secuelas en las personas que han sido víctimas del mismo, como la disminución de condiciones económicas, sociales y culturales favorables, el desplazamiento, la violencia sexual y múltiples afecciones en la salud mental, tales como ansiedad, depresión, dificultades para establecer relaciones interpersonales, agresividad, entre otros. Esta última, es relevante puesto que, al ser experimentada por los jóvenes víctimas del conflicto armado, favorece conductas externalizadas, y en lugar de disminuir la problemática de violencia en el país, se sigue reproduciendo. Objetivo: Describir los niveles y tipos de agresividad en adolescentes entre 12 y 17 años, víctimas y no víctimas del conflicto armado en Colombia, según sus condiciones socio-demográficas actuales. Método: Se tomó una muestra de 58 jóvenes entre 12 y 17 años; 22 víctimas y 36 no víctimas del conflicto armado, pertenecientes a una institución educativa. Se empleó el Test CAPI-A, (cuestionario de agresividad premeditada e impulsiva en adolescentes) y un breve cuestionario 
sociodemográfico. Resultados: Dentro de los resultados más relevantes, se encontró que hay mayor prevalencia de los diferentes tipos de agresividad en el grupo de los jóvenes no víctimas en comparación con el grupo de víctimas. Lo anterior pone de relieve múltiples factores externos e internos que pueden favorecer la agresividad, tales como un ambiente donde se convive con personas que presentan comportamiento violento y mensajes sociales que incitan a la agresividad.

Palabras claves: Conflicto armado en Colombia, violencia, agresividad, agresividad premeditada, agresividad impulsiva.

\section{Abstract}

Background: Colombia has been a country with numerous precedents of armed conflict, which has left great consequences in the people who have been victims of it, as the decrease of favorable economic, social and cultural conditions, displacement, sexual violence and multiple mental health problems, such as anxiety, depression, difficulties establishing interpersonal relationships, aggression, and so on. The last mentioned, is relevant since, when it is experienced by young victims of the armed conflict, it encourages externalized behaviors, and instead of reducing the problem of violence in the country, it keeps growing. Objective: Describing the levels and types of aggressiveness in adolescents between 12 and 17 years old, victims and non-victims of the armed conflict in Colombia, according to their current socio-demographic conditions. Method: A sample of 58 adolescents; between 12 and 17 years old, was taken; 22 victims and 36 non-victims of the armed conflict, belonging to one educative institution. A test CAPI-A (premeditated and impulsive aggressiveness questionnaire in adolescents) and a brief sociodemographic questionnaire was employed. Results: Among the most relevant results, it was found that there is a higher prevalence of the different types of aggression in the group of non-victim youths compared to the group of victims. This indicates the presence of multiple external and internal factors that may favor aggressiveness, such as an environment where people coexist with violent behavior and social messages that incite aggression.

Keywords: Armed conflict in Colombia, violence, aggressiveness, premeditated aggressiveness, impulsive aggressiveness. 


\section{Introducción}

Colombia ha sido históricamente un país afectado por la violencia. La guerra de la que ha sido testigo la población por más de 60 años, en su mayoría en espacios rurales, ha hecho que los habitantes de estos lugares, usualmente con bajo nivel económico y educativo, migren a las grandes ciudades, convirtiéndose en uno de los países con mayor índice de familias desplazadas y dejando más de 27.023 víctimas mortales (Centro Nacional de Memoria histórica, 2017). El desplazamiento forzado tiene como principales consecuencias, la desactivación de identidades, desarraigo, pérdida de la identidad social, generando a nivel psicológico efectos como temor y desesperanza y un comportamiento desestructurado o agitado en los niños (López-Navarrete et al., 2007; Andrade Salazar, 2010). El conflicto interno en Colombia ha dejado múltiples víctimas, entre ellas familias sumidas en la pobreza y desigualdad, niños y niñas sin padres ni cuidadores próximos, hombres y sobre todo mujeres víctimas de violencia sexual. La Corte Interamericana de Derechos Humanos (CIDH) ratificó los testimonios de las mujeres y niñas que fueron sometidas a esclavitud sexual, violadas por miembros de los grupos armados, obligadas a utilizar anticonceptivos y forzadas a abortar (CIDH, 2006), reafirmando la vulnerabilidad de las mujeres en escenarios de conflicto.

Hewitt, Gantiva, Vera, Cuervo y Hernández (2014) indican que ser testigo o víctima de violencia se relaciona "con problemas de conducta de tipo internalizado, evidenciados más en las niñas, tales como depresión, ansiedad y baja autoestima" (p.81).

El conflicto armado afecta en igual o mayor medida la salud mental de las personas que lo viven, para los niños, perder los padres o cuidadores tiene profundas repercusiones en su normal desarrollo, la exposición a eventos traumáticos que tienen relación con la guerra pueden verse reflejados en desordenes de la salud mental y que, además, perdurarían en el desarrollo de los niños y adolescentes (Hewitt et al., 2014).

El aumento de comportamientos violentos y agresivos es cada vez más común en adolescentes marcados por el conflicto armado. Entre las afectaciones psicológicas que presentan los niños y adolescentes como producto de la violencia se encuentran las dificultades en el desarrollo, problemas de atención, problemas cognitivos con ideas distorsionadas sobre lo ocurrido, culpa, vergüenza, inseguridad, indefensión, agresividad, pérdida de relaciones interpersonales o pérdida de confianza en el futuro, desarrollo de conductas regresivas, angustia por la separación de sus padres, miedo, ansiedad, depresión, trastornos del sueño, y labilidad emocional, entre otros (Hewitt et al., 2014). 
El desarrollo de los niños, niñas y adolescentes se puede ver afectado debido al desplazamiento forzado causado por la guerra. En su mayoría, estos niños han tenido experiencias con alguna forma de violencia que han generado traumas psicológicos y físicos, sentimientos de impotencia y rabia y una consecuente pérdida de la confianza, cambios en su estructura familiar, pérdida del entorno, disminución y casi desaparición de condiciones económicas favorables, culturales y sociales y finalmente agudización de la marginación y la pobreza (ACNUR, 2008).

Los efectos de la guerra en las comunidades van generalmente de la mano de desigualdades sociales, afectaciones en las relaciones sociales y formas de vinculación. Afectan seriamente la vida social, la salud mental, la salud integral y el bienestar; desintegran las redes de atención familiar y comunitaria; impiden el acceso a las necesidades básicas, la educación y la espiritualidad, aumentan las tasas de prevalencia de los trastornos mentales, incluyendo los del estado de ánimo (Hewitt et al., 2014, Sánchez Torres y Díaz, 2005; Minsalud, 2017).

La presencia de conductas agresivas en los adolescentes de nuestro país, posibilita el crecimiento de un círculo vicioso donde la violencia sólo trae más violencia y no da paso a la resiliencia ni a la configuración de estrategias de afrontamiento diferentes a las culturalmente aprendidas. Existen discusiones sobre si la agresividad es o no un comportamiento innato y, en algunas ocasiones, genético o aprendido y que está directamente relacionado con las condiciones sociales a las que el ser humano tiene acceso (Muñoz Prieto, 2009). Se han propuesto abordajes de la agresividad que explican su presencia y manifestación desde distinta procedencia. Entre estos están: aproximación evolucionista (etiología y socio biología), que apunta a una fuente biológica y adaptativa de la agresión; aproximación dinámica, que concibe la agresividad como expresión del instinto de muerte; modelo biológico que relaciona la agresividad con el efecto de hormonas y neurotransmisores en el organismo; la aproximación conductual, que interpreta la agresión como el resultado de condicionamiento por el ambiente; aproximación cognitiva según la cual la agresión es el resultado del afecto negativo producido por la experiencia de un suceso o acontecimiento desagradable, entre otros (Carrasco y González, 2006). Según algunos autores la agresividad aparece como posible efecto de las elaboraciones mentales y afectivas de las situaciones vividas, donde procesos intencionales y de significados, son capaces de activar conductas y sentimientos de ira que se sostienen involuntariamente (Cerezo, 2000/2002).

Existe en las víctimas del conflicto armado la sensación de impotencia y venganza por no haberse podido defender en aquel momento. Los impulsos 
agresivos aparecen en la víctima como la posibilidad de tomar un rol diferente al que tomaron al momento de ser víctima, es decir, una forma de compensar la indefensión a la que fue sometida; se enfrenta al otro con agresión frente a lo mínimo; hay acumulado demasiado rencor y rabia que deciden descargar por medio de agresiones (Aristizabal et al., 2012). Los estudios indican que la violencia relacionada con conflicto armado aumenta el riesgo de trastornos de ansiedad y agresividad en las victimas (Bell, Méndez, Martínez, Palma y Bosch, 2012; Hewitt et al., 2014).

La agresividad impulsiva es movida por la motivación momentánea de hacer daño y la activación emocional impensada de la mano de sentimientos como la ira, en ocasiones resultante de provocaciones percibidas en el otro, son principalmente la combinación que permite tomar malas decisiones. "La agresión impulsiva estaría relacionada con mecanismos de condicionamiento del miedo y el control afectivo, de ahí que ante la percepción de daño o dolor se halla automatizado la respuesta agresiva para evitar a través de esta conducta su cese" (Andreu Rodríguez, 2009, p.22). Las víctimas del conflicto armado, suelen reaccionar desde el miedo, utilizando la agresividad como mecanismo de defensa frente a la posibilidad de repetición (Aguilera Torrado, 2003).

Por otro lado, existe la agresividad premeditada que está motivada por el cumplimiento de objetivos, el mero hecho de dañar a una persona, lleva implícito un esfuerzo tanto físico como mental, pretende defender la coacción del otro, el poder o estatus social. "Es proactiva más que reactiva, ya que su meta principal no sería dañar a la víctima, sino lograr alguna otra consecuencia, como el acceso y control de recursos o beneficios" (Andreu Rodríguez, 2009, p.14). Obtener una recompensa psicológica, relacionada con historias de vida victimizadas puede significar un sentido de vida permeado por hacer daño al otro.

La violencia es uno de los fenómenos sociales más preocupantes, no solo por su prevalencia, sino por sus consecuencias en los niños, niñas y adolescentes del país. La legitimación de la violencia ha generado la normalización de las expresiones de agresividad, posibilitando en los jóvenes comportamientos y diferentes formas de violencia (Wallerstein, 2000; Padilla y Bermúdez, 2016). A partir de lo anterior es importante indagar por la presencia de agresividad en adolescentes víctimas del conflicto armado con el fin generar distintos programas de abordaje psicosocial de esta población para contrarrestar las consecuencias negativas para su futuro desarrollo. El presente estudio se dirige, de esta forma, a identificar los niveles y tipos de agresividad en una muestra de adolescentes víctimas y no víctimas del conflicto armado en Colombia según sus condiciones sociodemográficas. 


\section{Método}

Tipo de estudio: Investigación de enfoque cuantitativo, no experimental, transversal, de nivel descriptivo.

Participantes: En el estudio participaron estudiantes de una institución educativa que cuenta con la población estudiantil de afrodescendientes, indígenas, desplazados, víctimas del conflicto armado interurbano y/o rural o por desastres naturales. Se realizó un muestreo a conveniencia de 137 adolescentes con edades entre los 12 y los 17 años, quienes manifestaron su deseo de hacer parte de la investigación, de éstos, sólo 58 adolescentes completaron el consentimiento informado firmado por sus padres o acudientes, en la entrevista sociodemográfica 22 indicaron ser víctimas directas del conflicto armado.

Instrumentos: Con el fin de evaluar algunos aspectos importantes para la investigación, se diseñó un breve cuestionario en el cual se indagó por la edad, el sexo, el estrato socioeconómico, la configuración familiar y las vivencias del conflicto armado (ha sufrido la muerte de familiares, asesinados por miembros de grupos armados, se ha desplazado del lugar donde vivía de manera forzada por miembros de grupos armados y ha sido agredido de manera física o psicológica por miembros de grupos armados).

Para la medición de variables de agresividad se empleó el Test CAPI-A (Cuestionario de agresividad premeditada e impulsiva en adolecentes), diseñado y validado en población española por Andreu (2010), que evalúa la agresividad premeditada e impulsiva de los adolescentes de 12 a 17 años, tanto en población general como en población con diagnóstico clínico, de aplicación individual o colectiva con un tiempo de administración de 15 minutos. La prueba cuenta con dos escalas de valoración: premeditada e impulsiva. En general una puntuación percentil de 75 o superior en cualquiera de las dos escalas o en ambas es indicativa de agresividad impulsiva, premeditada o mixta. El Alfa de Cronbach para la escala de agresividad premeditada es de ,86 y de la escala de agresividad impulsiva es de, 82 .

Procedimiento: Con el fin de dar cumplimiento a la normatividad que rige la aplicación de pruebas con sujetos humanos, antes de iniciar el estudio se explicó a las directivas de la institución y a los estudiantes, el propósito, la metodología y los alcances de la investigación, posterior a ello se entregó el consentimiento informado para la firma de los padres y/o acudientes. El test fue aplicado de manera individual dentro de los salones de clase, sin límite de tiempo. 
Análisis de datos: Se utilizó la prueba estadística chi cuadrado para evaluar la asociación entre las variables de agresividad y las condiciones sociodemográficas de la población de estudio, estableciéndose un nivel de significancia estadística menor a 0,05. Los datos fueron procesos usando el programa SPSS versión 24.

\section{Resultados}

El mayor grupo de participantes fueron hombres. La edad promedio fue de 13 años $(\mathrm{DE}=2.5)$. El grado educativo del cual participó la mayor población fue décimo. Más de la mitad de los participantes pertenecen a estrato socioeconómico bajo. La mayoría de los hogares de los participantes están conformados por entre 3 y 5 personas. Por cada 10 participantes no víctimas hay 6 víctima del conflicto armado (ver tabla 1 ).

Tabla 1: Condiciones sociodemográficas

\begin{tabular}{llc}
\hline \multicolumn{1}{c}{ Variables } & Categorías & Frecuencia \% \\
\hline \multirow{2}{*}{ Sexo } & Mujer & $27(46,6)$ \\
& Hombre & $31(53,4$ \\
\hline \multirow{4}{*}{ Nivel educativo } & 6 & $14(24,1)$ \\
& 7 & $14(24,1)$ \\
& 8 & $3(5,2)$ \\
& 9 & $7(12,1)$ \\
& 10 & $16(27,6)$ \\
\multirow{4}{*}{ Estrato socioeconómico } & 11 & $4(6,9)$ \\
\hline \multirow{4}{*}{ Número de personas en el hogar } & Muy Bajo & $2(3,4)$ \\
& Bajo & $31(53,4)$ \\
& Medio-Bajo & $23(39,7)$ \\
& Medio & $2(3,4)$ \\
\hline \multirow{2}{*}{ Grupo } & 2 & $1(1,7)$ \\
& 3 & $14(24,1)$ \\
& 4 & $18(31,0)$ \\
& 6 & $14(24,1)$ \\
& 7 & $6(10,3)$ \\
& Víctima & $22(37,9)$ \\
\hline
\end{tabular}

Fuente: Elaboración propia 
Se identificó una mayor prevalencia de los diferentes tipos de agresividad en el grupo de las no víctimas en comparación con el grupo de víctimas. En cuanto al nivel de sinceridad, los participantes víctimas fueron predominantemente poco sinceros. Sin embargo, no hubo diferencias estadísticamente significativas entre los grupos (ver tabla 2).

Tabla 2: Índices de los tipos de agresividad y sinceridad

\begin{tabular}{|c|c|c|c|c|c|}
\hline \multicolumn{2}{|c|}{ Tipo de agresividad } & \multicolumn{2}{|c|}{ Grupo } & \multirow[t]{2}{*}{ Chi cuadrado } & \multirow[t]{2}{*}{ Valor $\mathbf{P}$} \\
\hline & & $\begin{array}{l}\text { Víctima } \\
\text { N (\%) }\end{array}$ & $\begin{array}{c}\text { No víctima } \\
\mathrm{N}(\%)\end{array}$ & & \\
\hline \multicolumn{2}{|c|}{ Premeditada } & $2(9,1)$ & $9(25,0)$ & 2,249 & 0,178 \\
\hline \multicolumn{2}{|l|}{ Impulsiva } & $7(31,8)$ & $16(44,4)$ & 0,910 & 0,340 \\
\hline \multicolumn{2}{|l|}{ Mixta } & $1(4,5)$ & $7(19,4)$ & 2,549 & 0,139 \\
\hline \multirow{2}{*}{ Sinceridad } & Más sincero & $9(40,9)$ & $18(50,0)$ & 0,454 & 0,501 \\
\hline & Poco sincero & $13(59,1)$ & $18(50,0)$ & & \\
\hline
\end{tabular}

Fuente: Elaboración propia

El grupo de mujeres tanto víctimas como no víctimas presentaron mayores niveles de agresividad premeditada y mixta, en comparación con los hombres. Con respecto a la agresividad impulsiva, los hombres del grupo de víctimas mostraron una ligera tendencia a este tipo de agresividad, en comparación con las mujeres del mismo grupo. Pese a lo anterior, no hay diferencias significativas en cuanto a los tipos de agresividad entre hombres y mujeres tanto víctimas como no víctimas (ver tabla 3 ).

Tabla 3: Tipos de agresividad según el sexo

\begin{tabular}{|c|c|c|c|c|c|}
\hline \multicolumn{2}{|c|}{ Tipo de agresividad } & \multicolumn{2}{|c|}{$\begin{array}{c}\text { Sexo } \\
\text { N (\%) } \\
\end{array}$} & \multirow[t]{2}{*}{ Chi cuadrado } & \multirow[t]{2}{*}{ Valor $\mathbf{P}$} \\
\hline & & Mujer & Hombre & & \\
\hline \multirow{2}{*}{ Premeditada } & Víctima & $2(100,0)$ & $0(0,0)$ & 3,178 & 0,156 \\
\hline & No víctima & $5(55,6)$ & $4(44,4)$ & 0,148 & 0,700 \\
\hline \multirow{2}{*}{ Impulsiva } & Víctima & $3(42,9)$ & $4(57,1)$ & 0,016 & 0,899 \\
\hline & No víctima & $10(62,5)$ & $6(37,5)$ & 1,800 & 0,315 \\
\hline \multirow{2}{*}{ Mixta } & Víctima & $1(100,0)$ & $0(0,0)$ & 1,513 & 0,219 \\
\hline & No víctima & $4(57,1)$ & $3(42,9)$ & 0,177 & 0,674 \\
\hline
\end{tabular}

Fuente: Elaboración propia 
El grupo de víctimas que cursa los grados noveno y décimo mostró mayor nivel de agresividad impulsiva. Por otro lado, el grupo de no víctimas mostró mayores niveles en los diferentes tipos de agresividad durante los dos primeros años cursados en segundaria. Sin embargo, no hubo diferencias estadísticamente significativas entre los grupos según el nivel educativo (ver tabla 4).

Tabla 4: Tipos de agresividad según el nivel educativo

\begin{tabular}{|c|c|c|c|c|c|c|c|c|c|}
\hline \multirow{2}{*}{\multicolumn{2}{|c|}{$\begin{array}{c}\text { Tipo de } \\
\text { agresividad }\end{array}$}} & \multicolumn{6}{|c|}{$\begin{array}{c}\text { Nivel educativo } \\
\text { N (\%) }\end{array}$} & \multirow[t]{2}{*}{$\begin{array}{c}\text { Chi } \\
\text { cuadrado }\end{array}$} & \multirow[t]{2}{*}{$\begin{array}{c}\text { Valor } \\
\mathbf{P}\end{array}$} \\
\hline & & Sexto & Séptimo & Octavo & Noveno & Décimo & Once & & \\
\hline \multirow{2}{*}{ 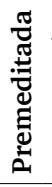 } & Víctima & $1(50,0)$ & $0(0,0)$ & $0(0,0)$ & $1(50,0)$ & $0(0,0)$ & $0(0,0)$ & 1,949 & 0,856 \\
\hline & No víctima & $2(22,2)$ & $3(33,3)$ & $1(11,1)$ & $0(0,0)$ & $3(33,3)$ & $0(0,0)$ & 2,207 & 0.820 \\
\hline \multirow{2}{*}{ 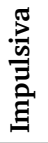 } & Víctima & $1(14,3)$ & $0(0,0)$ & $0(0,0)$ & $3(42,9)$ & $3(42,9)$ & $0(0,0)$ & 5,603 & 0,347 \\
\hline & No víctima & $4(25,0)$ & $5(31,3)$ & $1(6,3)$ & $0(0,0)$ & $5(31,3)$ & $1(6,3)$ & 2,421 & 0,788 \\
\hline \multirow{2}{*}{ 㞼 } & Víctima & $0(0,0)$ & $0(0,0)$ & $0(0,0)$ & $1(100,0)$ & $0(100,0)$ & $0(100,0)$ & 3,562 & 0,614 \\
\hline & No víctima & $3(42,9)$ & $2(28,6)$ & $0(0,0)$ & $0(0,0)$ & $2(28,6)$ & $0(100,0)$ & 4,037 & 0,544 \\
\hline
\end{tabular}

Fuente: Elaboración propia

Las personas no víctimas de estrato socioeconómico medio-bajo presentan mayor tendencia en agresividad premeditada, impulsiva y mixta en comparación con las no victimas en los otros estratos. En contraste, las personas víctimas de estrato bajo presentan mayor prevalencia de los diferentes tipos de agresividad, en comparación con las victimas en los otros estratos. Aunque no hubo diferencias estadísticamente significativas entre los grupos según el estrato socioeconómico (ver tabla 5).

Tabla 5: Tipos de agresividad según el estrato socioeconómico

\begin{tabular}{|c|c|c|c|c|c|c|c|}
\hline \multicolumn{2}{|c|}{ Tipo de agresividad } & \multicolumn{4}{|c|}{$\begin{array}{c}\text { Estrato socioeconómico } \\
\mathrm{N}(\%) \\
\end{array}$} & \multirow[t]{2}{*}{$\begin{array}{c}\text { Chi } \\
\text { cuadrado }\end{array}$} & \multirow[t]{2}{*}{ Valor $\mathbf{P}$} \\
\hline & & Muy Bajo & Bajo & Medio Bajo & Medio & & \\
\hline \multirow{2}{*}{ Premeditada } & Víctima & & $2(100,0)$ & $0(0,0)$ & $0(0,0)$ & 1,027 & 0,598 \\
\hline & No víctima & $1(11,1)$ & $1(11,1)$ & $7(77,8)$ & $0(0,0)$ & 6,373 & 0.095 \\
\hline \multirow{2}{*}{ Impulsiva } & Víctima & & $6(85,7)$ & $1(14,3)$ & $0(0,0)$ & 1,564 & 0,457 \\
\hline & No víctima & $1(6,3)$ & $6(37,5)$ & $9(56,3)$ & $0(0,0)$ & 1,635 & 0,652 \\
\hline \multirow{2}{*}{ Mixta } & Víctima & & $1(100,0)$ & $0(0,0)$ & $0(0,0)$ & 0,489 & 0,783 \\
\hline & No víctima & $0(0,0)$ & $1(14,3)$ & $6(85,7)$ & $0(0,0)$ & 5,229 & 0,156 \\
\hline
\end{tabular}

Fuente: Elaboración propia 
Del grupo de víctimas, quienes están en hogares compuestos por 5 personas, presentan la mayor tendencia en todos los tipos de agresividad. Mientras que, del grupo de no víctimas, quienes se encuentran en hogares compuestos por 3 personas, presentan la mayor tendencia a los diferentes tipos de agresividad. No obstante, no hubo diferencias estadísticamente significativas entre los grupos según el número de personas en el hogar (ver tabla 6).

Tabla 6. Tipos de agresividad según el número de personas en el hogar

\begin{tabular}{|c|c|c|c|c|c|c|c|c|c|}
\hline \multicolumn{2}{|c|}{ Tipo de agresividad } & \multicolumn{6}{|c|}{$\begin{array}{c}\text { Número de personas en el hogar } \\
\text { N (\%) }\end{array}$} & \multirow[t]{2}{*}{$\begin{array}{c}\text { Chi } \\
\text { cuadrado }\end{array}$} & \multirow[t]{2}{*}{$\begin{array}{c}\text { Valor } \\
\mathbf{P} \\
\end{array}$} \\
\hline & & 2 & 3 & 4 & 5 & 6 & 7 & & \\
\hline \multirow[t]{2}{*}{ Premeditada } & Víctima & & $0(0,0)$ & $0(0,0)$ & $2(100,0)$ & $0(0,0)$ & $0(0,0)$ & 5,867 & 0,209 \\
\hline & No víctima & $0(0,0)$ & $5(55,6)$ & $2(22,2)$ & $2(22,2)$ & $0(0,0)$ & $0(0,0)$ & 4,727 & 0,450 \\
\hline \multirow[t]{2}{*}{ Impulsiva } & Víctima & & $2(28,6)$ & $0(0,0)$ & $3(42,9)$ & $1(14,3)$ & $1(14,3)$ & 6,251 & 0,181 \\
\hline & No víctima & $1(6,3)$ & $8(50,0)$ & $4(25,0)$ & $2(12,5)$ & $1(6,3)$ & $0(0,0)$ & 7,742 & 0,171 \\
\hline \multirow[t]{2}{*}{ Mixta } & Víctima & & $0(0,0)$ & $0(0,0)$ & $1(100,0)$ & $0(0,0)$ & $0(0,0)$ & 2,794 & 0,593 \\
\hline & No víctima & $0(0,0)$ & $4(57,1)$ & $1(14,3)$ & $2(28,6)$ & $0(0,0)$ & $0(0,0)$ & 4,369 & 0,498 \\
\hline
\end{tabular}

Fuente: Elaboración propia

\section{Discusión}

En primera instancia, se halló que los participantes, según lo mostro el test CAPI, fueron predominantemente poco sinceros, lo que podría ser explicado por la conceptualización de la deseabilidad social, entendida siendo ésta comprendida como una necesidad de aprobación social (Lara-Cantu \& Suzan-Reed, 1988). Es decir, los participantes respondieron al cuestionario en función de lo que creían no iba a ser castigado socialmente. La deseabilidad social presenta un aspecto que debe tenerse en cuenta en las evaluaciones, ya que reduce la fiabilidad y validez de los resultados arrojados en algunas investigaciones debido a la necesidad de la persona de mostrar una imagen favorable de sí misma (Lara-Cantu \& Suzan-Reed, 1988).

En el estudio se encontró que los participantes no víctimas del conflicto obtuvieron mayores puntuaciones en todos los tipos de agresividad en comparación con los víctimas del conflicto; lo que difiere con los hallazgos encontrados en otras investigaciones, tales como la desarrollada por Hewwit et al. (2014), donde se encontró que los niños y jóvenes participantes víctimas del conflicto, presentaron un alto grado de agresión y conductas externalizadas (donde se incluyen conductas de agresión y conductas delictivas). De la misma manera, 
Aristizábal et al. (2012) concluyeron en su estudio realizado con 36 víctimas y victimarios del conflicto armado, los cuales se encontraban desplazados y reintegrados en Santa Marta y Barranquilla, que 4 sujetos víctimas del conflicto presentaban fallas en el control de impulsos agresivos relacionados con los efectos de la violencia, es decir, se les dificultaba manejar sentimientos como la ira y la venganza, afectando sus vínculos sociales y familiares.

Los mayores puntajes en agresividad obtenidos por los participantes no víctimas, podría ser explicado debido al ambiente en el cual se desenvuelven. Por ejemplo, Hewwit et al. (2014) resaltan que los efectos de la guerra en las comunidades van de la mano de desigualdades y afectaciones en los múltiples ámbitos de la persona: social, personal, psicológico, emocional, entre otros; disminuyendo la salud integral y el bienestar de éste. Es decir, aunque los participantes que mostraron mayores niveles de agresividad no hacían parte del conflicto armado, podrían hacer parte de otra guerra presente en el ambiente en el cual se encuentran: territorios con alta frecuencia de enfrentamientos, bandas criminales, padres violentos, entre otras; o dicho en las palabras de Cerezo (2000/2002), "la agresividad aparece como posible efecto de las elaboraciones mentales y afectivas de las situaciones vividas, donde procesos intencionales y de significados, son capaces de activar conductas y sentimientos de ira que se sostienen involuntariamente" (p.16).

En este aspecto, los resultados de la presente investigación coinciden con las conclusiones de Bell et al. (2012), en un estudio realizado con personas que vivían en zonas de conflicto armado, no hallaron una prevalencia de la agresión en alguno de los dos grupos estudiados, sino que pudieron concluir que la agresividad se hallaba presente tanto en las personas que estaban relacionadas con el conflicto, como en aquellas que no lo estaban.

Pasando a otro resultado, la investigación anteriormente mencionada, realizada por Hewwit et al. (2014), afirma que en el grupo de adolescentes víctimas del conflicto, ser de sexo masculino es un factor de riesgo (95\%) para agresión. Sin embargo, en la actual investigación se halló que la mayoría de las participantes mujeres víctimas del conflicto armado, presentaron mayores niveles de agresividad premeditada y mixta.

Adicionalmente, estos autores mencionan las afectaciones del conflicto armado en las mujeres: "Ser testigo o víctima de violencia se ha vinculado con problemas de conducta de tipo internalizado, evidenciados más en las niñas, tales como depresión, ansiedad y baja autoestima. Se ha encontrado que la violación es el predictor más fuerte de estos problemas" (Hewwit et al., 2014, p.81). Por el contrario, en esta investigación se encontraron problemas de conducta exteriorizado: la agresividad premeditada y mixta, teniendo en 
cuenta que la agresividad premeditada puede permanecer interiorizada durante la planeación hasta la obtención de la recompensa.

De la misma manera, en la actual investigación se hallaron mayores niveles de agresividad premeditada y mixta en mujeres no víctimas del conflicto, lo cual difiere con una investigación realizada por Andreu y Peña (2016), donde se midió la agresividad impulsiva y premeditada por medio de la aplicación del CAPI-A en 825 adolescentes españoles, la cual permitió concluir que los varones obtuvieron mayores puntuaciones en la agresividad premeditada. Para esclarecer la idea anterior, se puede tener en consideración la perspectiva neurobiológica ofrecida por la investigación realizada por Matthies et al. (2012), donde se encontró que las mujeres agresivas tenían un menor volumen de la amígdala (16-18\%) que aquellas clasificadas como no agresivas. De manera general, es importante señalar que las personas que se caracterizan por poseer una agresividad premeditada, suelen ser personas expuestas a modelos agresivos en su infancia (Andreu, 2010).

Con respecto a las puntuaciones en agresión impulsiva, los hombres víctimas del conflicto armado, obtuvieron mayores puntajes en comparación con las mujeres del mismo grupo. Este resultado es respaldado por la afirmación anteriormente mencionada, realizada por Hewwit et al. (2014), en el grupo de niños y adolescentes víctimas del conflicto, ser de sexo masculino es un factor de riesgo. A su vez, introducen una investigación realizada por Mels (2012) que concuerda con sus resultados, en la cual se menciona que los varones están más expuestos que las mujeres a la violencia, aumentándose la gravedad de la misma con la edad (Hewwit et al., 2014).

De la misma manera, hay varias investigaciones que aunque no fueron realizadas con víctimas del conflicto, concluyeron que los hombres obtuvieron mayores puntajes en agresividad impulsiva. Dentro de estas investigaciones, se encuentra la realizada por Andreu y Peña (2016) y la realizada por Ybañez (2014) con 525 adolescentes peruanos; ambas aplicaron el CAPI-A. Además, se encuentra la investigación realizada por Morales (2014) con 100 estudiantes universitarios de Ecuador, donde se midió la impulsividad por medio de la escala de impulsividad de Barrat, arrojando mayores puntajes los varones.

Asimismo, es relevante señalar que la agresividad impulsiva es ocasionada por una percepción constante y distorsionada de amenaza (Andreu y Peña, 2016), es decir, se percibe al otro como hostil. Estos sesgos cognitivos suelen tener su raíz en la victimización y malos tratos en la infancia (Andreu, 2010); por lo que en su desarrollo posterior, las víctimas del conflicto armado, tenderán a reaccionar desde el miedo, utilizando la agresividad como mecanismo de defensa frente a la posibilidad de repetición. 
Por último, se halló que no existe relación significativa entre el grado de escolaridad, el estrato socioeconómico, el número de personas en el hogar y las conductas agresivas. Sin embargo, existen diferentes autores que sostienen que la juventud es un factor de riesgo en la agresividad. Recalcando lo anterior, Castañeda y Rodríguez (2016) señalan que la agresividad se acentúa en la juventud debido a un escaso control de emociones. Del mismo modo, el aumento de comportamientos violentos y agresivos es cada vez más común en adolescentes marcados por el conflicto armado (Hewitt et al., 2014).

No se puede olvidar que la violencia, la agresión y el conflicto armado son multifactoriales, es decir, son el resultado de diversas variables como el ambiente externo (país, barrio, colegio), las interacciones que establece la persona con diferentes actores (padres, figuras de apego, amigos) y personales (factores biológicos, psicológicos y motivacionales). "Son tantos los factores relacionados con este comportamiento que parece que casi cualquier factor puede elicitar, de alguna manera, agresividad en la adolescencia" (Penado, 2012, p.70).

La violencia es uno de los fenómenos sociales más preocupantes en Colombia, más que por su prevalencia, por sus consecuencias en los niños, niñas y adolescentes del país. La legitimación de la violencia ha generado la normalización de las expresiones de agresividad, posibilitando en los jóvenes comportamientos y diferentes formas de violencia; legitimación que se ha instaurado por una larga historia de desplazamientos y grupos forzados y que se ha perpetuado a través de los medios de comunicación.

\section{Conclusiones}

Se encontró que los participantes no víctimas del conflicto obtuvieron mayores puntuaciones en todos los tipos de agresividad en comparación con las víctimas del conflicto. Estos resultados difirieron con diversas investigaciones donde se halló que los jóvenes víctimas del conflicto tenían una predisposición a la agresividad y conductas externalizadas, debido a fallas en el control de impulsos. Asimismo, ponen de relieve múltiples factores externos e internos que pueden favorecer la agresividad, tales como un ambiente donde se convive con personas que presentan comportamiento violento y mensajes sociales que incitan a la agresividad.

En la actual investigación se halló que la mayoría de las participantes mujeres víctimas del conflicto armado, presentaron mayores niveles de agresividad premeditada y mixta. Estos resultados difieren del mismo modo, con diversas investigaciones donde se encontró que el hombre era más 
propenso a presentar conductas agresivas. De esta manera, se puede concluir que las mujeres víctimas participantes de este estudio, poseen problemas de conducta externalizados como consecuencia de la exposición a la violencia.

Respecto a las puntuaciones en agresión impulsiva, los hombres víctimas del conflicto armado, obtuvieron mayores puntajes en comparación con las mujeres del mismo grupo; resultados respaldados por múltiples investigaciones, donde se esclarece que el hombre que está más expuesto a la violencia, es más impulsivo físicamente y al ser víctima del conflicto armado, puede hacer uso de la agresividad impulsiva como mecanismo de defensa para evitar la repetición de la misma.

Se halló también que no existe relación significativa entre el grado de escolaridad, el estrato socioeconómico, el número de personas en el hogar y las conductas agresivas. Sin embargo, otras investigaciones han encontrado que la juventud es un factor de riesgo para la agresividad, debido al escaso control de las emociones.

Finalmente, se puede concluir que los participantes fueron predominantemente poco sinceros, debido probablemente a la deseabilidad social, lo que puede disminuir la validez y confiabilidad de los resultados. Lo anterior, podría explicar por qué la mayoría de los resultados obtenidos en esta investigación difieren de los hallazgos presentados en otras investigaciones.

\section{Limitaciones}

A lo largo del proceso, se hallaron diferentes dificultades. Dentro de ellas, se puede resaltar la baja participación por parte de los jóvenes, debido a que de 123 estudiantes seleccionados a conveniencia, únicamente 58 decidieron participar del estudio.

De la misma manera, la participación de los jóvenes se vio dificultada por la ausencia de los consentimientos informados firmados por sus padres.

Además, como ya se mencionó anteriormente, la poca sinceridad por parte de los estudiantes fue una limitación, debido a la disminución de la validez de los resultados obtenidos.

\section{Referencias}

ACNUR (Agencia de la ONU para los refugiados). (2008). El ACNUR y la protección de los desplazados internos. Recuperado de: http://www.acnur.org/a-quien-ayuda/ desplazados-internos/ 
Aguilera Torrado, A. (2003). Las secuelas emocionales del conflicto armado para una política pública de paz. Convergencia. Revista de Ciencias Sociales, 10 (31): 11-37, https://www.redalyc.org/pdf/105/10503102.pdf

Andrade Salazar, J. A. (2010). Mujeres, niños y niñas, víctimas mayoritarias del desplazamiento forzado. Orbis. Revista Científica Ciencias Humanas, 6(16): 28-53, Recuperado de: https://www.redalyc.org/pdf/709/70916426003.pdf

Andreu, J. (2010). CAPI-A. Cuestionario de agresividad premeditada e impulsiva en adolescentes. Madrid: Editorial TEA Ediciones.

Andreu, J., Peña, M. (2016). Evaluación psicológica de la agresividad en adolescentes a través del cuestionario de agresividad premeditada e impulsiva. Ediciones ACIPE. Pp. 1717-1723. Recuperado de https://rua.ua.es/dspace/bitstream/10045/64086/1/ Psicologia-y-educacion_205.pdf

Andreu Rodríguez, J. (2009). Propuesta de un modelo integrador de la agresividad impulsiva y premeditada en función de sus bases motivacionales y sociocognitivas. Psicopatología clínica, legal y forense, 9: 85-98. Recuperado de: http:// masterforense.com/pdf/2009/2009art5.pdf

Aristizabal, E., Palacio, J., Madariaga, C., Osman, H., Parra, L. H., Rodríguez, J., López, G. (2012). Síntomas y traumatismo psíquico en víctimas y victimarios del conflicto armado en el caribe colombiano. Psicología desde el Caribe, 29(1): 123-152. Recuperado de: http://www.scielo.org.co/scielo.php?script=sci arttext\&pid $=$ s0123-417x2012000100008\&lng $=$ en\&tlng.

Bell, V., Méndez, F., Martínez, C., Palma, P. P., \& Bosch, M. (2012). Characteristics of the Colombian armed conflict and the mental health of civilians living in active conflict zones. Conflict and health, 6(10): 1-8, https://doi.org/10.1186/17521505-6-10

Castañeda, O., Rodríguez, M. (2016). Diferencias en los tipos de agresividad en adolescentes de colegio nacional y particular de la ciudad Cajamarca 2015 (Tesis de pregrado). Universidad Privada del Norte, Cajamarca, Perú. Recuperado de http://repositorio.upn.edu.pe/handle/11537/10763

Carrasco, M., González, M. J. (2006). Aspectos conceptuales de la agresión: definición y modelos explicativos. Acción Psicológica, 4 (2) pp. 7-38, Disponible en https:// www.redalyc.org/pdf/3440/344030758001.pdf

Centro Nacional de Memoria Histórica (2017). Informe General, iBasta Ya!. Recuperado de: http://www.centrodememoriahistorica.gov.co/basta-ya-menudestacado-informes 
Cerezo, F. (2000/ 2002). Bull- S. Test de Evaluación de la Agresividad entre Escolares. Madrid/ Bizkaia: Albor-Cohs. Recuperado de: http://www.gac.com.es/editorial/ INFO/Manuales/bullMANU.pdf

CIDH (Corte Interamericana de Derechos Humanos). (2006). Las mujeres frente a la violencia y la discriminación derivadas del conflicto armado en Colombia. Washington. Recucerado de: http://www.cidh.org/countryrep/colombiamujeres06sp/ informe\%20mujeres\%20colombia\%202006\%20espanol.pdf

Hewitt, N., Gantiva, C., Vera, A., Cuervo, M., Hernández, N., Juárez, F., Parada, A. (2014). Afectaciones psicológicas de niños y adolescentes expuestos al conflicto armado en una zona rural de Colombia. Acta Colombiana de Psicología, 17(1): 7989. doi:10.14718/Acp.2014.17.1.9

López-Navarrete, G., Perea-Martínez, A., Loredo, A., Trejo-Hernández, J., JordánGonzález, N. (2007). Niños en situación de guerra. Acta Pediátrica Mexicana, 28(2): 74 - 80. Recuperado de: http://www.redalyc.org/pdf/4236/423640304006.pdf.

Matthies, S., Rüsch, N., Weber, M., Lieb, K., Philipsen, A., Tuescher, O., Ebert, D., Hennig, J., van Elst, L. (2012). Small amygdala-high aggression? The role of the amygdala in modulating aggression in healthy subjects. World J Biol Psychiatry, 13(1):75-81. doi: 10.3109/15622975.2010.541282.

Mels, C. (2012). Entre pobreza y violencia: ¿cómo afectan los estresores diarios y la violencia a adolescentes en zonas de guerra? Ciencias Psicológicas, 6(2): 111-122. Recuperado de: http://www.scielo.edu.uy/scielo.php?pid=S1688$12012000200002 \&$ script $=$ sci_arttext\&tln.

MINSALUD (2017). Consecuencias del conflicto armado en la salud en Colombia. Informe técnico, 9. Disponible en https://www.ins.gov.co/Direcciones/ONS/ Informes/9\%20Consecuencias $\% 20$ del $\% 20$ Conflicto $\% 20$ Armado $\% 20$ en $\% 20$ la\%20Salud\%20en\%20Colombia.pdf

Morales, R. (2014). Impulsividad y agresividad en los estudiantes de la universidad técnica de Ambato. Congreso Iberoamericano de Ciencia, Tecnología, Innovación y Educación. 1-17. Recuperado de: https://www.oei.es/historico/congreso2014/ memoriactei/688.pdf

Muñoz Prieto, M. (2009). Incidencia del acoso escolar en alumnos de $4^{\circ}$ y $6^{\circ}$ de Educación Primaria en colegios de la ciudad de Vigo. [Tesis doctoral], Universidad de Salamanca. Recuperado de: https://gredos.usal.es/jspui/bitstream/10366/76544/1/DPETP_ Munoz_Prieto_MM_Incidencia_del_acoso.pdf

Lara-Cantú, A., Suzan-Reed, M. (1988) La escala de deseabilidad social de Marlowe y Crowne: un estudio psicométrico. Salud Mental. 11(3): 25-29. Recuperado de: http://hdl.handle.net/123456789/1573 
Padilla, A., Bermúdez, A. (2016). Normalizar el conflicto y desnormalizar la violencia: retos y posibilidades de la enseñanza crítica de la historia del conflicto armado colombiano. Revista Colombiana de Educación, 71: 219-251, http://www.scielo. org.co/pdf/rcde/n71/n71a09.pdf

Penado, M. (2012). Agresividad reactiva y proactiva en adolescentes: efectos de los factores indiviuales y socio-contextuales. [Tesis doctoral]. Universidad Complutense de Madrid, España. Recuperado de http://studylib.es/doc/6569071/agresividadreactiva-yproactiva-en-dolescentes---e

Sánchez Torres, F., Díaz, A. (2005). Los efectos del conflicto armado en el desarrollo social colombiano, 1990-2002. PNUD y del Ministerio de la Protección Social. Disponible en https://economia.uniandes.edu.co/assets/archivos/Documentos CEDE/d2005-58.pdf

Wallerstein, N. (2000). Violencia en Colombia: reflexiones de una profesora visitante. Revista Facultad Nacional de Salud Pública, 18 (2): 101-115, https://www.redalyc. org/pdf/120/12018208.pdf

Ybañez, J. (2014). Agresividad premeditada e impulsiva en adolescentes de Florencia de Mora- Trujillo- La Libertad. Cátedra Villareal, 2(2): 29-39. Recuperado de http://revistas.unfv.edu.pe/index.php/RCV/article/viewFile/34/34 